\title{
Order of report and processing in tachistoscopic recognition'
}

\author{
A. O. DICK ${ }^{2}$ ANO D. J. K. MEWHORT \\ UNIVERSITY OF WATERLOO
}

Two groups of 12 Ss were shown tachistoscopically 4 letters and 4 numbers. With in these alpha-numeric sequences, three variables were systematically manipulated: grouping of items, initial item, and familiarity of the letter sequences. One group of $S$ s reported first letters then numbers from each sequence; the other group reported numbers then letters. The results indicated that grouping, redundancy, and order of report had significant effects on letter accuracy but not on number accuracy. These effects were interpreted in terms of processing, particularly the use of spatial and identity information during recognition.

In the typical tachistoscopic recognition experiment, subjects are required to identify several items presented simultaneously. In such experiments, the accuracy of identification depends on the order of report; material reported early in the response sequence is more likely to be correct than that reported late in the response sequence (Bryden, 1960; Mewhort, 1966). Such data have been interpreted in terms of the interaction of sequential report with rapid memory loss (e.g., Bryden, 1960). According to this interpretation, the subject reports the material sequentially but forgets some of it before report can occur. It may be, however, that this relation is not a function of ordered report but reflects the way in which material is processed during recognition.

Several experiments suggest that the recognition of alphabetic material involves a sequential operator. Probably this sequential operator develops as the subject learns to read. For example, Bryden (1960) presented both letters and forms tachistoscopically and required subjects to report the material in either a left-to-right or a right-to-left direction. The results indicated that subjects can report in a right-to-left direction more easily for forms than for letters. Furthermore, the subjects reported that they had to think of the letters in a left-to-right direction before they could report in a right-to-left direction. This was not necessary for forms. Such data suggest that recognition of letters involves unique directional processes. Other data suggest that these unique left-to-right processes use both the identity and spatial information available for each letter. For example, more letters can be identified from a familiar letter sequence than from an unfamiliar one (Mewhort, 1966; Miller, Bruner, \& Postman, 1954). In their demonstration of this fact, Miller et al (1954) utilized the stable pattern of letter usage in English in order to construct pseudo-words of various degrees of familiarity or levels of approximation to English. In such materials the familiarity of the familiar sequences depends on a sequential analysis of letters. Furthermore, Mewhort (1966) has shown that the effective familiarity of the familiar sequences depends on the spacing of the letters. Such data indicate that the processes sensitive to the familiarity of letter sequences must take into account the relative spatial information as well as the identity of the letters. Reading experience teaches us that the letter is not the unit because letters are usually integrated into words. Such integration requires that the subject notice the relative position of each letter.

Although data parallel to those available for letters is not available for numbers, it seems reasonable to suppose that somewhat different processes are involved. Letter usage in English exhibits stable sequential dependencies, but no such dependencies exist for numbers. If this sort of argument is correct, the left-to-right processes which apply to letters as a result of reading experience would not apply to numbers.

The present experiment was designed to test the suggestion that the relation between accuracy and order of report can be explained in terms of an interaction of sequential report with rapid memory loss. The logic of the experiment is based on the assumption that letter material is normally processed in a left-to-right order but that numbers are not. Subjects were shown both letters and numbers simultaneously but the order of report was determined by instruction. If the relation normally found between the position in the response sequence and accuracy of report results from the interaction of sequential report with rapid memory loss, the forced order of report would exaggerate this relation. However, if this relation were a matter of sequential processing rather than sequential report, the forced order of report should have a greater effect on material that is normally processed in a fixed order. In addition, if sequential processing is involved, the arrangement and order of the materials in the stimulus sequence ought to be important.

\section{Subjects}

\section{METHOD}

The Ss were 24 undergraduates taking an intro- 
ductory course in psychology; participation fulfilled part of the course requirement.

\section{Materials}

Six sets of 24 stimulus cards were prepared. On each card, four letters and four numbers were arranged in a row. All 26 letters of the alphabet and the digits 1-9 were used; "zero" was not used because of the confusability with the letter "O." The stimulus cards were prepared with Letraset Instant Lettering Style 287 using upper case letters. Each item was approximately $1 / 4 \mathrm{in}$. high and $1 / 8 \mathrm{in}$. wide. The overall angle subtended by the stimulus was $1.0 \times 4.0$ degrees. Within each set of cards, three variables were systematically manipulated: (1) half of the letter sequences were taken from 4-order approximations to English, the other half of the letter sequences from 0-order approximations (Miller, Bruner, \& Postman, 1954); (2) the letters were grouped all together, alternated in pairs with numbers, or alternated singly with numbers; $(3)$ the initial item in the sequence was either a letter or a number. Each $S$ received two presentations of each of the 12 stimulus conditions. The six sets of stimuli comprised a means for controlling potential differences between alpha-numeric sequences; each sequence was used in each set. However, across the six sets, the arrangement and grouping of each sequence was systematically varied.

\section{Procedure}

The $24 \mathrm{Ss}$ were randomly divided into two groups defined by two instruction conditions. Half of the Ss were asked to report the material by giving the letters first and then the numbers, while the other half were required to report numbers first, then letters. Two Ss in each group were presented one of the six sets of stimuli. The materials were presented in a random order in a Gerbrands mirrortype tachistoscope at a duration of $100 \mathrm{msec}$. A small black fixation point was presented in one field midway between Items 4 and 5 ; the test stimuli were presented in the other field. The luminance of the fixation field was $3.8 \mathrm{ft}-\mathrm{L}$ while the luminance of the exposure fleld was about $3.5 \mathrm{ft}-\mathrm{L}$. The fixation field was illuminated continuously except when the exposure field was illuminated.

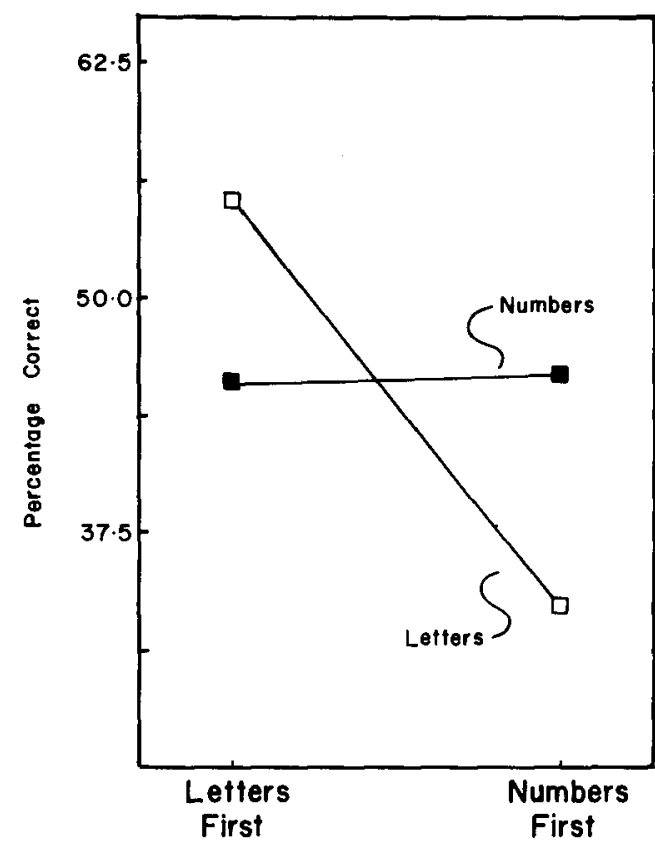

Fig. 1. Percent items correct as a function of the order of report.

A preliminary study demonstrated that the Ss could easily perform the task required; indeed, in the present experiment, when the Ss were asked to report letters first, on only seven of 288 trials was the order of report confused. In three of these cases, the confusion resulted because the $S$ reported the number "zero" rather than the letter "O."

\section{RESULTS}

For each trial, total correct responses for letters and for numbers were scored separately without regard to stimulus position. The data summarized in Table 1 were obtained by averaging the two replications within each condition.

The order of report had no effect on the identification of numbers $(F=0.72, d f=1 / 12$; Fig. 1$)$, but had a large effect on the identification of letters $(F=33.21, d f=1 / 12, p<0.005 ; F i g .1)$. In other words, the number data showed no effect of output interference. In addition, order of report interacted with familiarity of the letter sequences. The difference between the two levels of familiarity was greater when the

Table 1. Mean number of items correct in each condition

\begin{tabular}{|c|c|c|c|c|c|c|c|c|c|c|c|c|}
\hline \multirow{3}{*}{$\begin{array}{l}\text { Fomiliority } \\
\text { Stimulus Order } \\
\text { Grouping }\end{array}$} & \multicolumn{6}{|c|}{ O-order } & \multicolumn{6}{|c|}{ 4-order } \\
\hline & & L-N & & & $N-L$ & & & L-N & & & $N-L$ & \\
\hline & 4 & 2 & 1 & 4 & 2 & 1 & 4 & 2 & 1 & 4 & 2 & 1 \\
\hline \multicolumn{13}{|l|}{ Letters Correct } \\
\hline Numbers first & 1.33 & 1.46 & 1.33 & 1.54 & 1.16 & 1.37 & 1.54 & 1.46 & 1.08 & 1.50 & 1.41 & 0.87 \\
\hline Letters first & 2.33 & 1.87 & 1.91 & 2.50 & 1.79 & 2.04 & 2.71 & 2.46 & 2.08 & 2.62 & 2.04 & 2.04 \\
\hline \multicolumn{13}{|l|}{ Numbers Correct } \\
\hline Numbers first & 1.66 & 1.75 & 1.50 & 1.79 & 2.04 & 1.96 & 1.91 & 1.83 & 1.79 & 1.87 & 2.00 & 1.91 \\
\hline Letters first & 1.66 & 1.66 & 1.96 & 1.91 & 1.91 & 1.66 & 1.87 & 1.91 & 1.83 & 1.83 & 1.75 & 1.83 \\
\hline
\end{tabular}




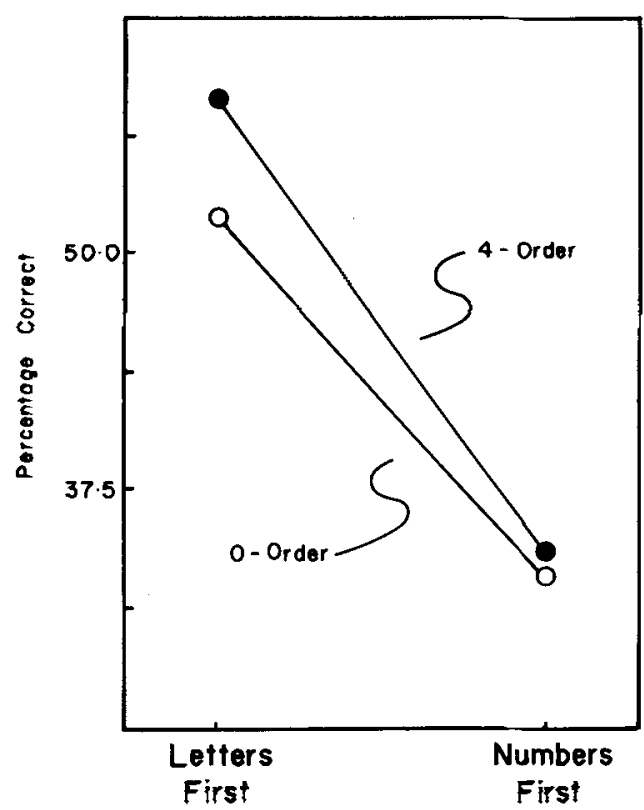

Fig. 2. Percent letters correct for both levels of tamiliarity as a function of the order of report.

letters were reported before the numbers $(F=3.85$, $\mathrm{df}=1 / 12, \mathrm{p}<0.10$; Fig. 2).

The fact that order of report was not important for the identification of numbers but had a large effect on letter identification rules out the suggestion that the usual relation between order and accuracy of report is a function of an interaction of sequential report with rapid memory loss. If this suggestion were correct, then reporting the letters first ought to have greatly lowered recognition performance for numbers. Rather, these data suggest that the critical

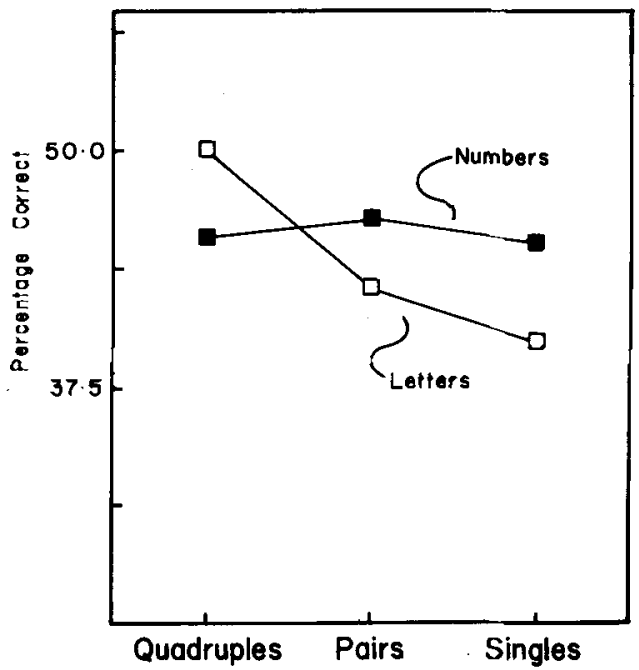

Fig. 3. Percent items correct as a function of the grouping of the materials.

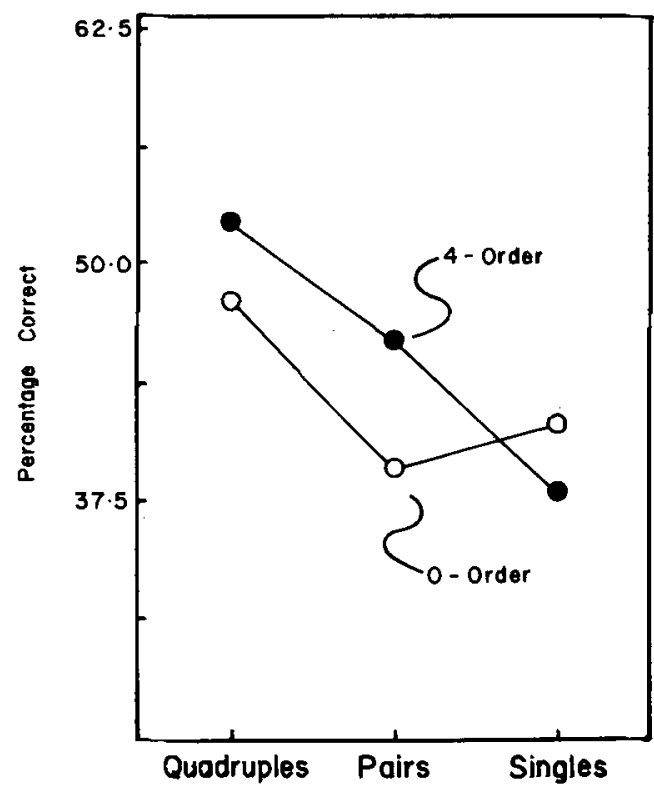

Fig. 4. Percent letters correct for both levels of familiarity as a function of the grouping of the materials.

interaction concerns processing with rapid memory loss.

The grouping of the items had a large effect on letter accuracy $(F=11.21, d f=2 / 24, p<0.005$; Fig. 3$)$; however, number accuracy was not significantly different across the three groupings $(F=0.21$, df $=2 / 24$; Fig. 3). For letters, recognition was better for familiar materials unless the letters and the numbers were alternated $(F=5.22$, df $=2 / 24, p<0.05$; Fig. 4). Separating the letters by numbers had the effect of destroying the familiarity of the 4-order sequence.

Finally, variation of the initial item had no significant effect. Furthermore, there were no significant interactions involving this variable. Of particular interest is the comparison of accuracy for four letters-four numbers with four numbersfour letters. On the assumption that the $S$ scans the material in a strict left-to-right order, one would expect that it would be easier to report the letters first if they were in fact first in the stimulus. This was not the case.

\section{DISCUSSION}

The present data show that: (1) the recognition of letters is better the closer they are together; (2) letter recognition is better if the response is immediate; (3) number recognition is not the same as letter recognition. Neither grouping of items nor the order of report had any significant effect on number recognition.

A recent experiment (Dick, 1967a) provides a 
basis for explaining the present data. In that experiment, Ss were shown both Ietters and numbers arranged in two rows. A post-exposure auditory cue was used to instruct the $S$ to report either letters or numbers or, alternately, either the top row or the bottom row. The materials were arranged so that over several trials the material to be reported was the same regardless of whether the auditory cue referred to an identity discrimination (letters vs numbers) or to a spatial one (top vs bottom row). By delaying the post-exposure cue, it was shown that the identity information remained available much longer than the spatial information. The present data, as well as those reported by Mewhort (1966), show that the relative spatial information is necessary for the integration of letter sequences. In view of the fact that the familiarity of the 4-order materials depends upon sequential (1.e, spatial) information, this result is not difficult to accept. According to Dick (1967a), delaying the report causes rapid loss of usable spatial information. In this light, the difference between letter and number recognition for the two orders of report (Fig. 1) is interpretable in terms of the spatial information avallable. Assuming that such a spatial information is not necessary for numbers, the order of report instruction would not affect number accuracy. This assumption is consistent with the present data (Fig. 3), which show no effect of grouping on number recognition.

Two final points should be made. First, it might be argued that the two groups of Ss used different strategies as a result of knowing in advance how they were to report the material. In some situations it is true that differential strategies are possible. For example, when Ss are requested to report by spatial location there is a dramatic difference between pre-exposure and post-exposure report instructions. In such a case the $S$ is able to move his eyes to the appropriate position when he knows in advance what he is to report. However, when Ss are asked to report by identity class such an effect is not obtained (Dick, 1967b). In the present experiment, the presentation sequence was randomized, hence, $S$ did not know in advance where the materials would be; he only knew what to do first. On the basis of existing data, then, differential strategies are unlikely. Second, a fixed sequential processing system is not a function of familiarity per se, as Bryden $(1960,1967)$ has suggested, but rather is a function of past experience with the particular material to be reported. Numbers, while highly familiar, display more flexible processing than letters. Thus, the present experiment suggests a technique for studying processing mechanisms; comparisons of letter and number data should yield additional information about processing mechanisms.

\section{References}

Bryden, M. P. Tachistoscopic recognition of non-alphabetic material. Canad. J. Psychol., 1960, 14, 78-86.

Bryden, M.P. A model for the sequential organization of behaviour. Canad. J. Psychol., 1967, 21, 37-56.

Dick, A. O. Short-term memory for several stimulus attributes. Paper presented at the Meeting of the East. Psychol. Assoc., Boston, 1967a.

Dick, A. O. Visual injormation processing and the memory trace. Unpublished Ph.D. Thesis, University of Waterloo, 1967b.

Mewhort, D. J. K. Sequential redundancy and letter spacing as determinants of tachistoscopic recognition. Canad. J. Psychol, $1966,20,435-444$.

Miller, G. A., Bruner, J. S., \& Postman, L. Familiarity of letter sequences and tachistoscopic identification. J. gen. Psychol., $1954,50,129-139$.

\section{Notes}

1. This research was supported in part by Defence Research Board of Canada Grant No. 9401-26 to M. P. Bryden. The authors wish to thank M. P. Bryden for his encouragement and assistance in connection with this research. Some of the data in this paper were presented at the Meetings of the Canadian Psychological Association, Ottawa, 1967, with reversed authorship.

2. Now at Lake Forest College, Lake Forest, Illinois.

(Accepted for publication July 31, 1967.) 